\title{
Solving Ordinary Differential Equations with Range Conditions. Applications*
}

\author{
Enrique Castillo ${ }^{\dagger}$ \\ Antonio J. Conejo \\ Carmen Castillo§ \\ Roberto Mínguez
}

\begin{abstract}
This paper introduces the problem of solving ordinary differential equations with extra linear conditions written in terms of ranges, and deals with the corresponding existence and uniqueness problems. Some methods for analyzing the existence of solutions and obtaining the set of all solutions, based on the theory of cones and polyhedra, are given. These solutions are found by first converting the problem to a system of linear algebraic equations and then applying the corresponding well-known theory for solving and discussing the existence and uniqueness of solutions of these systems. Finally, the methods are illustrated by their application to some practical examples of the beam problem.
\end{abstract}

Key words. range extra conditions, beams, cones, polyhedra, uniqueness, existence theorem

AMS subject classifications. $15 \mathrm{~A} 03,15 \mathrm{~A} 39,34 \mathrm{~A} 12$

DOI. $10.1137 / \mathrm{S} 003614450444470 \mathrm{X}$

I. Introduction. When dealing with ordinary differential equation (ODE) problems one normally looks for unique solutions, so that the differential equation must be complemented with some extra conditions (initial, boundary, etc.) such that the infinite set of functions associated with the solutions of the differential equation is so restricted that only one of these functions satisfies the extra conditions (see, for example, Boyce and DiPrima [1] and Coddington and Levinson [8]). This is the standard paradigm in the field of differential equations, where uniqueness has played an outstanding role and has been the concern of many researchers.

For example, consider a problem that is defined by the linear ODE of order $n$ in standard form:

$$
\frac{d^{n} x}{d t^{n}}+a_{1}(t) \frac{d^{n-1} x}{d t^{n-1}}+\cdots+a_{n-1}(t) \frac{d x}{d t}+a_{n}(t) x=h(t) .
$$

*Received by the editors June 29, 2004; accepted for publication (in revised form) April 15, 2005; published electronically May 2, 2006. This work was supported by the Spanish Ministry of Science and Technology (projects DPI2002-04172-C04-02, DPI2003-01362), the Spanish Ministry of Education, and the Fulbright Program.

http://www.siam.org/journals/sirev/48-2/44470.html

${ }^{\dagger}$ Department of Applied Mathematics and Computational Sciences, University of Cantabria, Avda. Castros s/n, 39005 Santander, Spain (castie@unican.es).

${ }^{\ddagger}$ Department of Electrical Engineering, University of Castilla-La Mancha, Avda. Camilo José Cela s/n, 13071 Ciudad Real, Spain (Antonio.Conejo@uclm.es).

$\S$ Department of Civil Engineering, University of Castilla-La Mancha, Avda. Camilo José, Cela s/n, 13071 Ciudad Real, Spain (MariaCarmen.Castillo@uclm.es).

IDepartment of Applied Mathematics, University of Castilla-La Mancha, 13071 Ciudad Real, Spain (Roberto.Minguez@uclm.es). 
The general solution to problem (1) is of the form

$$
x(t)=\sum_{i=1}^{n} c_{i} \phi_{i}(t)+\psi_{p}(t)
$$

where $c_{1}, c_{2}, \ldots, c_{n}$ are arbitrary constants, the set of functions $\left\{\phi_{1}(t), \phi_{2}(t), \ldots, \phi_{n}(t)\right\}$ is a fundamental system of solutions for the corresponding homogeneous equation, and $\psi_{p}(t)$ is a particular solution to problem (1).

This implies that (1) has infinitely many solutions which can be characterized by the set of constants $c_{1}, c_{2}, \ldots, c_{n}$.

In order to reduce this set of solutions one can, for example, consider the extra linear set of conditions

$$
\sum_{j=1}^{s} d_{j} x^{\left(r_{i j}\right)}\left(t_{i j}\right)=b_{i}, \quad i=1,2, \ldots, \ell,
$$

where $d_{j} \in \mathbb{R}, j=1,2, \ldots, s, x^{\left(r_{i j}\right)}\left(t_{i j}\right)$ refers to the $r_{i j}$ th derivative of $x(t)$ at $t=t_{i j}$, and $b_{i} \in \mathbb{R}, i=1,2, \ldots, \ell$.

In particular, one can choose the $\psi_{p}(t)$ function in $(2)$ to be the solution to problem (1) with the homogeneous conditions

$$
\sum_{j=1}^{s} d_{j} x^{\left(r_{i j}\right)}\left(t_{i j}\right)=0, \quad i=1,2, \ldots, \ell
$$

which implies

$$
\sum_{j=1}^{s} d_{j} \psi_{p}^{\left(r_{i j}\right)}\left(t_{i j}\right)=0, \quad i=1,2, \ldots, \ell .
$$

Conditions (3) restrict the set of possible solutions, and, if adequately chosen, they lead to a unique solution, i.e., to a particular subset of constants $\left\{c_{1}, c_{2}, \ldots, c_{n}\right\}$ in (2).

However, in practice, users are frequently unable to give conditions (3) with fixed numbers (the $b_{i}$ constants), and they prefer to define the extra conditions in a more imprecise way, e.g., providing intervals or ranges. For example, in engineering one cannot assure fixed values and one has to deal with different assumptions, so that ranges or intervals are much more appropriate than fixed values to define the $b_{i}$ parameters. It is worth mentioning that these problems have important applications in practically all fields and do not necessarily lead to uniqueness of solutions.

When fixed values $b_{i}, i=1,2, \ldots, \ell$, for the extra conditions are used, $n$ (the order of the differential equation) independent conditions can be given; otherwise, the problem may have no solution (for $\ell>n$ ). However, when ranges replace fixed values, the number of extra conditions can be unlimited. This makes the existence and uniqueness theorems, and the methods for obtaining solutions, very different from the standard ones. Consequently, new methods are required.

The main original contributions of this paper are as follows: (a) to introduce (to our knowledge) for the first time the problem of solving ODEs with range linear conditions as an interesting and useful alternative to the standard practice of fixed conditions, (b) to give methods for solving this problem, i.e., for obtaining the set of all solutions, and (c) to explain how the existence of a solution and its uniqueness can 
be tested. In addition, the proposed methods are illustrated by their application to one practical example.

In this paper we assume that the functional space to which $x(t)$ belongs is adequately chosen so that the required regularity conditions hold, and thus our problems are well posed and we can concentrate on the range problem.

The paper is structured as follows. Section 2 states the differential equation problem in terms of ranges or intervals. Section 3 shows how the set of all solutions can be obtained. Section 4 discusses the existence and uniqueness problems. In section 5 an application to beams is described. Finally, in section 6, some conclusions are given.

2. Statement of the Problem of Differential Equations with Range Conditions. In this section we analyze what happens if we replace the point conditions in (3) by interval conditions; i.e., our new problem can be defined by the linear ODE

$$
\frac{d^{n} x}{d t^{n}}+a_{1}(t) \frac{d^{n-1} x}{d t^{n-1}}+\cdots+a_{n-1}(t) \frac{d x}{d t}+a_{n}(t) x=h(t),
$$

and linear conditions of the type

$$
\left\{b_{i}^{\text {lower }} \leq \sum_{j=1}^{s} d_{j} x^{\left(r_{i j}\right)}\left(t_{i j}\right) \leq b_{i}^{\text {upper }}, i=1,2, \ldots, \ell\right\},
$$

where $\left(b_{i}^{\text {lower }}, b_{i}^{\text {upper }}\right)$ are the intervals replacing the constant values $b_{i}$, and $\ell$ does not need to equal $n$.

Before dealing with solving the problem (6)-(7), we assume that we have already determined a fundamental system of solutions $\left\{\phi_{1}(t), \phi_{2}(t), \ldots, \phi_{n}(t)\right\}$ using wellknown methods.

3. Solving the Problem. Since the general solution of (6) is of the form (2), we must have

$$
b_{i}^{\text {lower }} \leq \sum_{j=1}^{s}\left(d_{j} \sum_{i=1}^{n} c_{i} \phi_{i}^{\left(r_{i j}\right)}\left(t_{i j}\right)\right)+\sum_{j=1}^{s} d_{j} \psi_{p}^{\left(r_{i j}\right)}\left(t_{i j}\right) \leq b_{i}^{\text {upper }}, \quad i=1,2, \ldots, \ell,
$$

and by (5) and interchanging the sums, we get

$$
b_{i}^{\text {lower }} \leq \sum_{i=1}^{n}\left(c_{i} \sum_{j=1}^{s} d_{j} \phi_{i}^{\left(r_{i j}\right)}\left(t_{i j}\right)\right) \leq b_{i}^{\text {upper }}, i=1,2, \ldots, \ell,
$$

which is a system of linear inequalities in the $c_{i}$ 's.

As the general solution of a complete system of linear equations can be written as a particular solution plus a linear space, the general solution of a complete system of linear inequalities is a polyhedron and can be written as a linear space plus an acute cone (a cone $C$ is said to be acute if no vector $\mathbf{s} \in C$ satisfies $-\mathbf{s} \in C$ ) plus a polytope, i.e., in the form

$$
\left(\begin{array}{c}
c_{1} \\
c_{2} \\
\vdots \\
c_{n}
\end{array}\right)=\sum_{i=1}^{I} \rho_{i}\left(\begin{array}{c}
u_{1 i} \\
u_{2 i} \\
\vdots \\
u_{n i}
\end{array}\right)+\sum_{j=1}^{J} \pi_{j}\left(\begin{array}{c}
v_{1 j} \\
v_{2 j} \\
\vdots \\
v_{n j}
\end{array}\right)+\sum_{k=1}^{K} \lambda_{k}\left(\begin{array}{c}
w_{1 k} \\
w_{2 k} \\
\vdots \\
w_{n k}
\end{array}\right), \rho_{i} \in \mathbb{R}, \pi_{j} \geq 0, \lambda_{k} \geq 0
$$

where $\sum_{k=1}^{K} \lambda_{k}=1$. 
In other words, sets of vectors $\mathbf{u}, \mathbf{v}$, and $\mathbf{w}$ exist such that the general solution of (9) can be written as in (10), where the linear space is generated by the set of vectors $\left\{\mathbf{u}_{i}, i=1,2, \ldots, I\right\}$, the cone is generated by the set $\left\{\mathbf{v}_{j}, j=1,2, \ldots, J\right\}$, and the polytope is generated by the set $\left\{\mathbf{w}_{k}, k=1,2, \ldots, K\right\}$. Due to the unbounded, unbounded-from-below, and bounded character of the corresponding coefficients $\rho_{i}$, $\pi_{j}$, and $\lambda_{k}$, these linear space, cone, and polytope components play an important role in discussing the nature of all possible solutions.

The problem of obtaining the sets $\mathbf{u}, \mathbf{v}$, and $\mathbf{w}$, which depend on the $\mathbf{b}^{\text {upper }}, \mathbf{b}^{\text {lower }}$, and $\mathbf{d}$ vectors, the $\left(t_{i j}\right)$ matrix, and the fundamental system of solutions $\left\{\phi_{1}(t), \phi_{2}(t)\right.$, $\left.\ldots, \phi_{n}(t)\right\}$ is not trivial. Detailed methods for obtaining minimal sets of generators $\mathbf{u}_{i}, \mathbf{v}_{j}$, and $\mathbf{w}_{j}$ of this polyhedron can be seen in Padberg [9] and Castillo et al. $[4,2,3,7,5,6]$. The main idea of the methodology is as follows.

Solving the problem (6) with (7) is in fact merely a question of solving the system of linear inequalities $(9)$ in $c_{1}, c_{2}, \ldots, c_{n}$, which can be written as

$$
\begin{aligned}
-\sum_{i=1}^{n}\left(c_{i} \sum_{j=1}^{s} d_{j} \phi_{i}^{\left(r_{i j}\right)}\left(t_{i j}\right)\right)+b_{i}^{\text {lower }} c_{n+1} & \leq 0, \quad i=1,2, \ldots, \ell, \\
\sum_{i=1}^{n}\left(c_{i} \sum_{j=1}^{s} d_{j} \phi_{i}^{\left(r_{i j}\right)}\left(t_{i j}\right)\right)-b_{i}^{\text {upper }} c_{n+1} & \leq 0, \quad i=1,2, \ldots, \ell, \\
-c_{n+1} & \leq 0, \\
c_{n+1} & =1,
\end{aligned}
$$

where the artificial variable $c_{n+1}$ and the redundant constraint $\left(-c_{n+1} \leq 0\right)$ have been added for convenience and so $c_{n+1}$ must be removed later.

The solution of the system (11)-(13) is the dual cone $C$ of the cone generated by the vectors, the components of which are the row coefficients of this system. Standard methods for obtaining this dual cone can be seen in Padberg [9] and Castillo et al. $[4,2,3,7,5,6]$. This dual cone can be written in standard form, i.e., as the sum of a linear space plus an acute cone.

Once the dual cone has been obtained, $c_{n+1}$ is forced to be 1 (see (14)). In this step, the polytope part of the cone is obtained if some of the linear space and acute cone generators of the dual cone contain a nonnegative $n+1$ component. Finally, the artificial $(n+1)$ th component is removed, and the polyhedral set of all solutions of the form (10) is obtained. A detailed example is given in section 5.

Then, the set of solutions of the problem (6) with (7) is

$$
\begin{aligned}
x(t) & =\left(\begin{array}{llll}
\phi_{1}(t) & \phi_{2}(t) & \ldots & \phi_{n}(t)
\end{array}\right)\left(\begin{array}{c}
c_{1} \\
c_{2} \\
\vdots \\
c_{n}
\end{array}\right)+\psi_{p}(t) \\
& =\sum_{r=1}^{n} \psi_{r}(t)+\psi_{p}(t),
\end{aligned}
$$

where

$$
\psi_{r}(t)=\left(\sum_{i=1}^{I} \rho_{i} u_{r i}+\sum_{j=1}^{J} \pi_{j} v_{r j}+\sum_{k=1}^{K} \lambda_{k} w_{r k}\right) \phi_{r}(t), \quad r=1,2, \ldots, n,
$$

and $\rho_{i} \in \mathbb{R}, \pi_{j} \geq 0, \lambda_{k} \geq 0, \sum_{j=1}^{K} \lambda_{k}=1$. 
Equations (15) and (16) provide a method for obtaining the set of all solutions of the problem (6) with (7) if they exist. The method is illustrated in the example in section 5 .

4. Testing for Existence and Uniqueness. One interesting problem is that of existence and uniqueness of solution to the problem (6) with (7), which leads to the problem of existence and uniqueness of solution of the system of linear inequalities (9).

To analyze the existence problem, i.e., if this system is compatible (a solution to the problem (6) with (7) exists), one can proceed as follows. First, the system (9) is written as

$$
\begin{array}{r}
-\sum_{i=1}^{n}\left(\left(c_{i}^{*}-c_{0}^{*}\right) \sum_{j=1}^{s} d_{j} \phi_{i}^{\left(r_{i j}\right)}\left(t_{i j}\right)\right)+c_{n+i}^{*}=-b_{i}^{\text {lower }}, \quad i=1,2, \ldots, \ell, \\
\sum_{i=1}^{n}\left(\left(c_{i}^{*}-c_{0}^{*}\right) \sum_{j=1}^{s} d_{j} \phi_{i}^{\left(r_{i j}\right)}\left(t_{i j}\right)\right)+c_{n+\ell+i}^{*}=b_{i}^{\text {upper }}, \quad i=1,2, \ldots, \ell, \\
c_{i}^{*} \geq 0, \quad i=0,1, \ldots, 2 \ell+n,
\end{array}
$$

where we have replaced the real variables $c_{i}$ by the difference of two nonnegative variables $c_{i}^{*}$ and $c_{0}^{*}$, i.e., $c_{i}=c_{i}^{*}-c_{0}^{*}$.

The system (9) is compatible if there exist nonnegative $c_{i}^{*}$ 's such that (17)-(19) hold, but this means that the vector

$$
\mathbf{h}=\left(-b_{1}^{\text {lower }},-b_{2}^{\text {lower }}, \ldots,-b_{\ell}^{\text {lower }}, b_{1}^{\text {upper }}, b_{2}^{\text {upper }}, \ldots, b_{\ell}^{\text {upper }}\right)^{T}
$$

of its independent terms (in (17)-(18)) belongs to the cone generated by the columns of this system, i.e., if the dot products of $\mathbf{h}$ by the generators of the corresponding dual cone are nonpositive.

So, to test for existence, we proceed as follows:

1. The generators of the dual cone of the cone generated by the columns of the coefficient matrix of the system (17)-(18) are obtained.

2. The dot products of the vector $\mathbf{h}$ by all generators are calculated.

3. If at least one of the dot products is positive, the system is incompatible; otherwise, it is compatible and the initial problem (6) with (7) has a solution.

This method for testing the existence of a solution will be illustrated in section 5 .

The uniqueness problem leads to the uniqueness of a solution of the system (9) (see Castillo et al. [2, p. 218]), so that uniqueness holds if and only if the set of possible $c$ constants degenerates to a point (a polytope with a unique generator), i.e., if the cone $C$, solution of (11)-(13), is generated by a single vector.

5. Application to the Beam Problem. In this section the above method is illustrated by its application to the beam problem.

Consider a beam subject to a vertical load $p(t)$. It is well known that the differential equation for the beam is

$$
x^{(i v)}(t)=\frac{p(t)}{E I}, \quad 0 \leq t \leq L,
$$


where $x(t)$ is the deflection of the beam at point $t$, and $E$ and $I$, which are assumed to be constant, are the Young modulus and the moment of inertia of the cross section of the beam, respectively. To be specific, we let $p(t) /(E I)=-100$ and $L=1$.

Then, the general solution of (20) is of the form

$$
x(t)=\sum_{i=1}^{4} c_{i} \phi_{i}(t)+\psi_{p}(t) .
$$

The particular solution $\psi_{p}(t)$ in this case is the solution of (20) with the boundary conditions

$$
x(0)=x^{\prime}(0)=0, \quad x(1)=x^{\prime}(1)=0
$$

that leads to the particular solution

$$
\psi_{p}(t)=\frac{-25 t^{2}}{6}+\frac{25 t^{3}}{3}-\frac{25 t^{4}}{6}
$$

and

$$
\sum_{i=1}^{n} c_{i} \phi_{i}(t)=c_{1}+c_{2} t+c_{3} t^{2}+c_{4} t^{3}
$$

If the deflections and rotations at the two ends $(t=0$ and $t=1)$ of the beam are bounded, say, by -1 and 1 , we have

$$
\begin{aligned}
& -1 \leq x(0) \leq 1, \\
& -1 \leq x^{\prime}(0) \leq 1, \\
& -1 \leq x(1) \leq 1, \\
& -1 \leq x^{\prime}(1) \leq 1,
\end{aligned}
$$

and using (21) and (24) the following constraints are obtained:

$$
\left(\begin{array}{l}
-1 \\
-1 \\
-1 \\
-1
\end{array}\right) \leq\left(\begin{array}{llll}
1 & 0 & 0 & 0 \\
0 & 1 & 0 & 0 \\
1 & 1 & 1 & 1 \\
0 & 1 & 2 & 3
\end{array}\right)\left(\begin{array}{l}
c_{1} \\
c_{2} \\
c_{3} \\
c_{4}
\end{array}\right) \leq\left(\begin{array}{l}
1 \\
1 \\
1 \\
1
\end{array}\right)
$$

To solve this system of inequalities as in (11)-(14), an artificial unknown $c_{5}=1$ is used, so that the two systems in (29) become

$$
\left(\begin{array}{rrrrr}
1 & 0 & 0 & 0 & -1 \\
0 & 1 & 0 & 0 & -1 \\
1 & 1 & 1 & 1 & -1 \\
0 & 1 & 2 & 3 & -1 \\
-1 & 0 & 0 & 0 & -1 \\
0 & -1 & 0 & 0 & -1 \\
-1 & -1 & -1 & -1 & -1 \\
0 & -1 & -2 & -3 & -1
\end{array}\right)\left(\begin{array}{l}
c_{1} \\
c_{2} \\
c_{3} \\
c_{4} \\
c_{5}
\end{array}\right) \leq\left(\begin{array}{l}
0 \\
0 \\
0 \\
0 \\
0 \\
0 \\
0 \\
0
\end{array}\right)
$$

the solution of which is the cone

$$
\mathbf{c}=\mathbf{V} \pi
$$


where

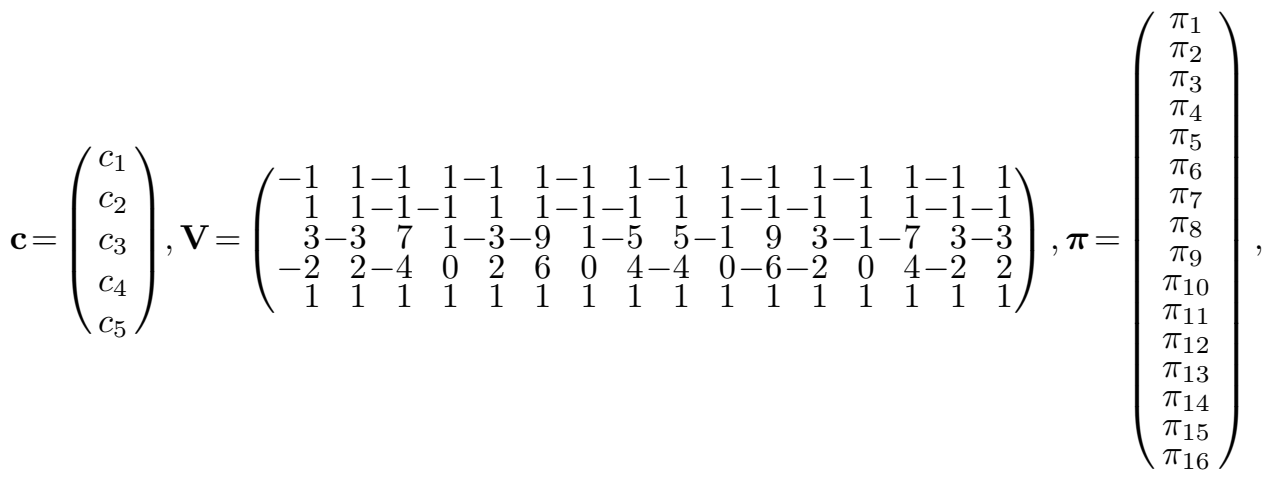

$$
\begin{aligned}
& \pi_{j}>0, j=1,2, \ldots, 16 \text {. }
\end{aligned}
$$

Note that $\mathbf{V}$ is the matrix whose columns are the vectors $\mathbf{v}$ in (10). Since $c_{5}$ must be forced to be 1 (see (14)), $\sum_{j=1}^{16} \pi_{j}=1$; i.e., the $\pi_{j}$ 's become $\lambda_{k}$ 's, i.e., the nonnegative linear combination in (31) becomes a linear convex combination.

For the polyhedron to have linear space and acute cone parts, the dual cone must also have linear space and acute parts and their generators must contain null and nonnull last components. Since in this particular case the cone (32) has no linear space part and the last components of all its generators are equal to 1, the polyhedron degenerates to a polytope.

Finally, removing the artificial unknown $c_{5}$, the general solution of (20) with (25)-(28) becomes

$$
x(t)=\left(1 t t^{2} t^{3} \psi_{p}(t)\right) \mathbf{V} \boldsymbol{\lambda}, \quad \lambda_{k}>0, k=1,2, \ldots, 16, \quad \sum_{k=1}^{16} \lambda_{k}=1 .
$$

The 16 generator functions, which allow all the corresponding possible solutions to be generated (as a polytope) and the associated bounds to be obtained, are shown in the upper left corner of Figure 1, where the particular solution $\psi_{p}(t)$ is also shown (dotted line). The remaining plots correspond to the first, second, and third derivatives, which correspond to rotations (upper right plot), bending moments (lower left plot), and shear forces (lower right plot). Note that from a practical point of view these derivatives of $x(t)$ are more relevant than the deflection $x(t)$, because they allow the defining of the steel reinforcement in the case of concrete beams and the checking of its strength for steel beams.

If an extra constraint is added, consisting of limiting to -1.2 the deflection of the beam at $t=1 / 2$ (i.e., the problem (20) with (25)-(28) and

$$
x(1 / 2) \geq-1.2
$$

is solved), Figure 2 shows the 21 generators of the new polytope of solutions, and the particular solution $\psi_{p}(t)$ (dotted line).

Finally, Figure 3 shows the resulting 14 generator functions if the shear force at $t=0$ is limited to 50 and the deflection constraint at $t=1 / 2$ is removed; i.e., those associated with the problem (20) with (25)-(28) and

$$
x^{\prime \prime \prime}(0)<50 .
$$



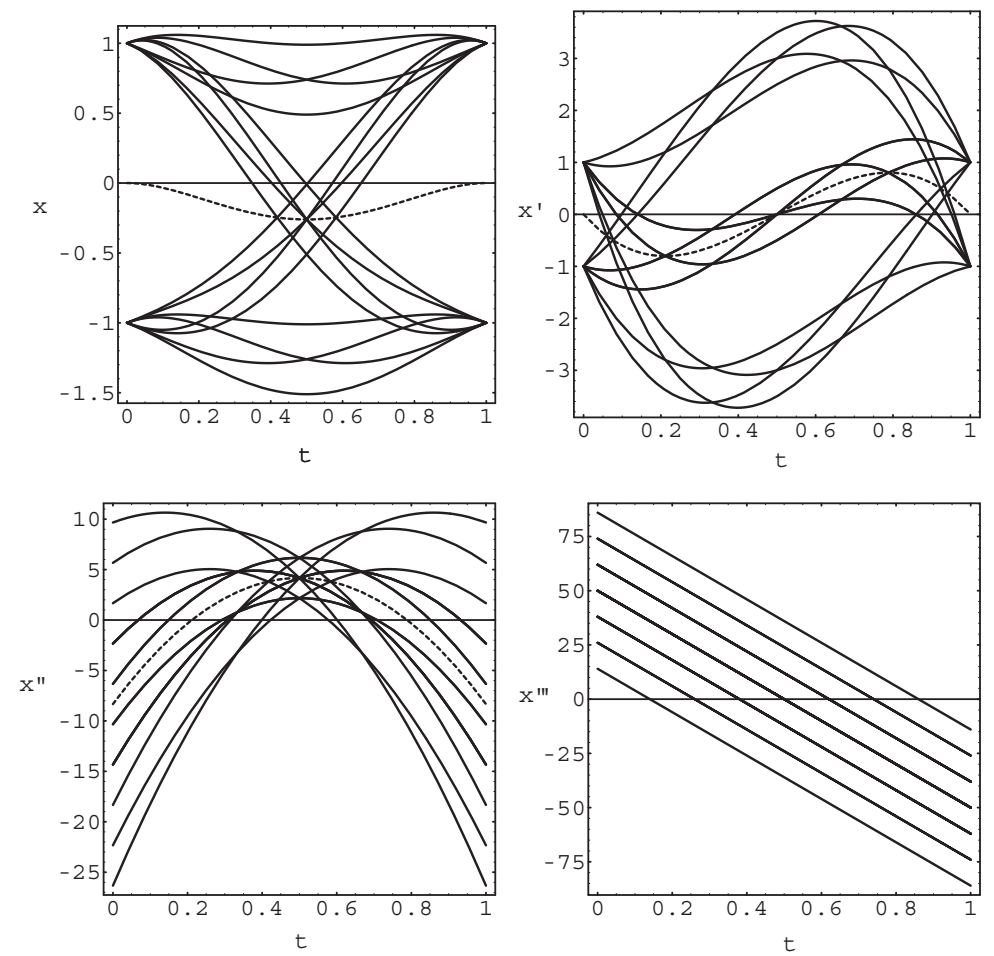

Fig. I Illustration of the generators of the polytope of all solutions for deflections, rotations, bending moments, and shear forces.

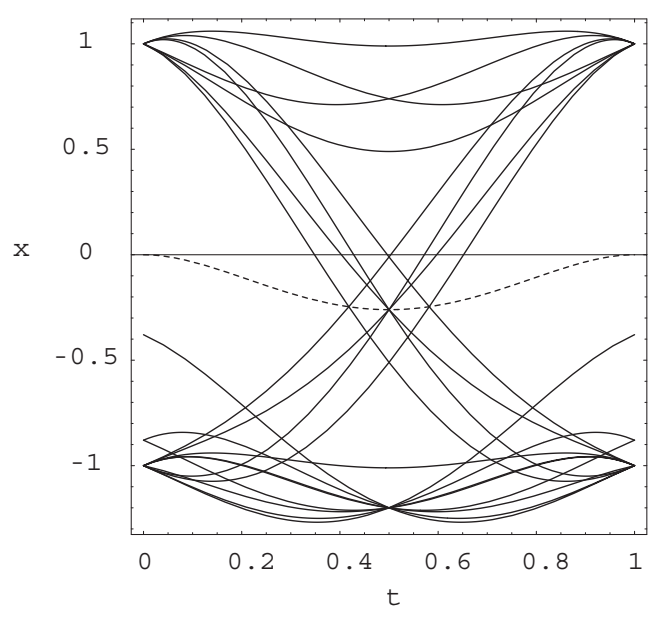

Fig. 2 Illustration of the 21 generators of the polytope of all solutions and a particular case (dotted line) if the deflection of the beam at $t=1 / 2$ is limited to -1.2 .

5.I. Existence of Solutions. To illustrate how the existence method described in section 4 can be applied, two cases of parameterized constraints such as those in (25)(28) are considered and the conditions for the existence of a solution are determined. 

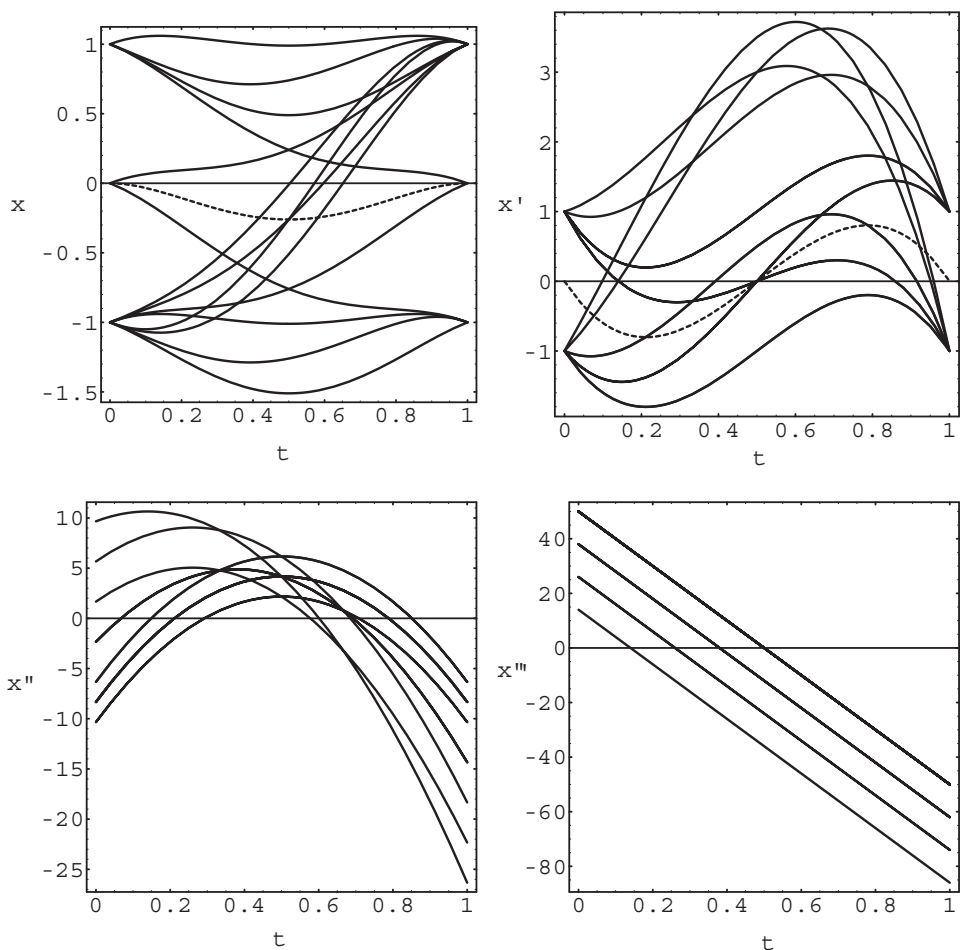

Fig. 3 Illustration of the 14 generators of the polytope of all solutions and a particular case (dotted line) for the deflections, rotations, bending moments, and shear forces, if the shear force at $t=0$ is limited to 50 .

Case 1. Consider the following set of constraints:

$$
\begin{gathered}
b_{1}^{\text {lower }} \leq x(0) \leq b_{1}^{\text {upper }}, \\
b_{2}^{\text {lower }} \leq x^{\prime}(0) \leq b_{2}^{\text {upper }}, \\
b_{3}^{\text {lower }} \leq x(1) \leq b_{3}^{\text {upper }}, \\
b_{4}^{\text {lower }} \leq x^{\prime}(1) \leq b_{4}^{\text {upper }} .
\end{gathered}
$$

In this case, the system (34)-(37) becomes

$$
\left(\begin{array}{rrrr}
1 & 0 & 0 & 0 \\
0 & 1 & 0 & 0 \\
1 & 1 & 1 & 1 \\
0 & 1 & 2 & 3 \\
-1 & 0 & 0 & 0 \\
0 & -1 & 0 & 0 \\
-1 & -1 & -1 & -1 \\
0 & -1 & -2 & -3
\end{array}\right) \quad\left(\begin{array}{c}
c_{1} \\
c_{2} \\
c_{3} \\
c_{4}
\end{array}\right) \leq\left(\begin{array}{c}
b_{1}^{\text {upper }} \\
b_{2}^{\text {upper }} \\
b_{3}^{\text {upper }} \\
b_{4}^{\text {upper }} \\
-b_{1}^{\text {lower }} \\
-b_{2}^{\text {lower }} \\
-b_{3}^{\text {lower }} \\
-b_{4}^{\text {lower }}
\end{array}\right)
$$

which, using the techniques explained in section 4 , leads to

$$
b_{i}^{\text {lower }} \leq b_{i}^{\text {upper }} \forall i,
$$

which are the well-known and obvious conditions for existence of solution. 
Case 2. If the constraint $x(1 / 2) \geq a$ is added to the constraints (34)-(37), the existence conditions become (39) together with

$$
4 b_{1}^{\text {upper }}+b_{2}^{\text {upper }}+4 b_{3}^{\text {upper }}-b_{4}^{\text {lower }}+8 a \geq 0,
$$

which is a symbolic expression. Since $a=-1.2$ and $b_{i}^{\text {upper }}=-b_{i}^{\text {lower }}=1$ in the second example in section 5 , the existence conditions are satisfied.

Case 3. If condition $x^{\prime \prime \prime}(0) \leq q$ is added to the conditions (34)-(37), the existence conditions become (39) together with the symbolic constraint

$$
-12 b_{3}^{\text {upper }}+12 b_{1}^{\text {lower }}+6 b_{2}^{\text {lower }}+6 b_{4}^{\text {lower }}-q+50 \leq 0 .
$$

Since $q=50$ and $b_{i}^{\text {upper }}=-b_{i}^{\text {lower }}=1$ in the third example in section 5 , the existence conditions are satisfied.

Note that for large enough $q$, condition (41) holds, and then these conditions are equivalent to conditions (39).

6. Conclusions. In this paper we have introduced for the first time the problem of differential equations with range constraints and justified how important it is in practical applications.

Within the context of solving ODEs with range constraints, we have introduced in this paper a general method for the following applications.

1. Testing the existence of solutions, which is, in fact, merely a question of finding the generators of the dual cone of a cone, calculating some dot products, and checking that they are nonpositive. In addition, the method makes it possible to use a parametric treatment; i.e., the set of symbolic constraints that the initial or boundary values must satisfy for the problem to have a solution can be easily obtained.

2. Obtaining the set of all solutions of an ODE, the conditions of which are given not by point values, but as ranges.

3. Determining whether or not the problem has a unique solution.

4. All the proposed methods reduce the initial problem to a system of linear inequalities, which is treated (discussed its existence and uniqueness of solutions, and solved it) using existing methods.

Finally, the method has been illustrated by its application to the beam problem, as an example of the many possible applications of the range constraints case in different fields of science and technology.

Acknowledgments. The authors want to express their gratitude to the editor, the referees, and María Eugenia Pérez for their constructive comments that certainly led to an improvement of the paper.

\section{REFERENCES}

[1] W. E. Boyce And R. C. DiPrima, Elementary Differential Equations and Boundary Value Problems, John Wiley and Sons, New York, 1997.

[2] E. Castillo, A. Cobo, F. Jubete, and R. E. Pruneda, Orthogonal Sets and Polar Methods in Linear Algebra: Applications to Matrix Calculations, Systems of Equations and Inequalities, and Linear Programming, John Wiley and Sons, New York, 1999.

[3] E. Castillo, A. Cobo, F. Jubete, R. E. Pruneda, and C. Castillo, An orthogonally based pivoting transformation of matrices and some applications, SIAM J. Matrix Anal. Appl., 22 (2000), pp. 666-681.

[4] E. Castillo, A. Conejo, P. Pedregal, R. García, and N. Alguacil, Building and Solving Mathematical Programming Models in Engineering and Science, Pure Appl. Math. (N.Y.), John Wiley and Sons, New York, 2001. 
[5] E. Castillo, M. Esquivel, And R. E. Pruneda, Automatic generation of linear programming problems for computer aided instruction, Internat. J. Math. Educ. Sci. Tech., 32 (2001), pp. 209-232.

[6] E. Castillo And F. Jubete, The $\gamma$-algorithm and some applications, Internat. J. Math. Educ. Sci. Tech., 35 (2004), pp. 369-389.

[7] E. Castillo, F. Jubete, E. Pruneda, and C. Solares, Obtaining simultaneous solutions of linear subsystems of equations and inequalities, Linear Algebra Appl., 346 (2002), pp. 131154.

[8] E. A. Coddington And N. Levinson, Theory of Ordinary Differential Equations, McGraw-Hill, New York, 1955.

[9] M. Padberg, Linear Optimization and Extensions, Springer-Verlag, Berlin, 1995. 